\title{
ZBTB7 evokes 5-fluorouracil resistance in colorectal cancer through the NF-kB signaling pathway
}

\author{
ZEXIN WANG, XILAN ZHAO, WEI WANG, YISHU LIU, YANYAN LI, JUNYONG GAO, \\ CANCAN WANG, MEIYU ZHOU, RUYAN LIU, GUOFA XU and QI ZHOU
}

Department of Oncology, Chongqing Fuling Central Hospital, Chongqing 408099, P.R. China

Received October 31, 2017; Accepted February 20, 2018

DOI: 10.3892/ijo.2018.4521

\begin{abstract}
Zinc finger and BTB domain containing 7A (ZBTB7), a POZ/BTB and Krüppel erythroid myeloid oncogenic factor, is critical for the tumorigenicity and progression of various cancer types. ZBTB7 has been reported to promote the cell proliferation of colorectal cancers (CRC). However, the function of ZBTB7 to 5-fluorouracil (5-FU) resistance has not yet been studied. In the current study, ZBTB7 expression and function in 5-FU resistance in CRC were investigated using with multidisciplinary approaches, including western blot analysis, Transwell assay, CCK8 and a tumor xenograft model. Overexpression of ZBTB7 was increased the level of proteins associated with cell invasion and epithelial-mesenchymal transition. ZBTB7 inhibition attenuated the invasion and enhanced the apoptosis of CRC cells. $\mathrm{IC}_{50}$ values and cell viability were significantly reduced in cells with short hairpin RNA (shRNA)-mediated ZBTB7 depletion compared with the control group. 5-FU administration decreased viability to a greater extent in the ZBTB7-shRNA group compared with the control, which was dose- and time-dependent. Analysis of gene expression omnibus data demonstrated that ZBTB7 mediated 5-FU resistance, potentially through nuclear factor $(\mathrm{NF})-\kappa \mathrm{B}$ signaling. NF- $\kappa \mathrm{B}$ inhibitor SN50 reversed ZBTB7-induced resistance in CRC. Collectively, the findings demonstrated that ZBTB7 mediated 5-FU resistance in CRC cells through NF- $\kappa \mathrm{B}$ signaling. Thus, targeting ZBTB7 and $\mathrm{NF}-\kappa \mathrm{B}$ signaling may be an effective strategy to reverse 5-FU resistance in CRC.
\end{abstract}

\section{Introduction}

Colorectal cancer (CRC) is a very common malignancy worldwide and is the third leading cause of cancer-associ-

Correspondence to: DrZexin Wang or ProfessorQiZhou,Department of Oncology, Chongqing Fuling Central Hospital, 2 Gaosuntang Road, Fuling, Chongqing 408099, P.R. China

E-mail:w_zexin@163.com

E-mail: qizhou112@163.com

Key words: zinc finger and BTB domain containing 7A, nuclear factor- $\kappa \mathrm{B}$, colorectal cancers, chemoresistance ated mortality (1). The survival rate of patients with CRC remains unsatisfactory, despite effective therapeutic strategies including surgical resection and chemo-radiotherapy. 5-Fluorouracil (5-FU), antimetabolite medication, is a fluorinated analog active against a wide range of solid tumors, including pancreatic cancer (2-4), biliary tract cancer (5), breast cancer $(6,7)$ and hepatocellular cancer $(8,9)$. 5 -FU-based therapy is one of the treatment regimens as the adjuvant or palliative chemotherapy for patients with CRC (10,11). 5-FU acts through various mechanisms, but principally as a thymidylate synthase inhibitor, eliciting cytotoxicity by interrupting the function of nucleotide synthetic enzyme thymidylate synthase. Additionally, it incorporates fluoronucleotides into RNA and DNA $(12,13)$. A subset of patients with CRC is primarily refractory or have acquired chemoresistance to 5-FU, which has poses a major challenge for clinical practice to improve therapeutic efficiency. Thus, investigation of the mechanisms and discovery of potential treatments targets for patients with CRC with 5-FU resistance is urgently required.

Zinc finger and BTB domain containing 7A (ZBTB7), is part of the POZ and Krüppel/zinc finger and BTB (POK) protein family (14). It is involved in pleiotropic functions and cellular differentiation. ZBTB7 physically interacts with other POK family members, such as B-cell lymphoma 6 proteins (15). ZBTB7, considered to be an oncogene, is overexpressed in a variety of cancer types, and involved in the pathogenesis of cancer (16), including lung cancer (17), prostate cancer (18), liver cancer (19) and oral cancer (20). ZBTB7 drives tumorigenesis as a master regulator and is required for the regulation of other tumor suppressor genes, such as p53 (21). ZBTB7 has also been documented to be an important regulator of CRC initiation and progression (22). ZBTB7 knockdown could inhibit cell proliferation, and induce cell cycle arrest and promote apoptosis independently of p14-E3 ubiquitin-protein ligase MDM2-p53 pathway (23).

Currently, there limited knowledge of the role of ZBTB7 in $\mathrm{CRC}$, and the specific mechanisms the mediate the oncogenic role of ZBTB7 in CRCs is unknown. To the best of our knowledge, no previous studied have investigated the association between of ZBTB7 expression in CRC and 5-FU resistance. In the present study, it was demonstrated that ZBTB7 knockdown sensitized the 5-FU response to in CRC, potentially via the nuclear factor $(\mathrm{NF})-\kappa \mathrm{B}$ signaling pathway. 


\section{Materials and methods}

Microarray data analysis. The genetic profiles of patients with CRC were acquired from the Gene Expression Omnibus (GEO; ncbi.nlm.nih.gov/geo/) data repository (GSE39582, GSE36133, GSE17538, GSE31595, GSE33113, GSE37892, GSE38832 and GSE39084). The method used for quality control and raw data processing was previously described (24).

Differences in ZBTB7 expression among multiple cancer types were assessed using data also from GEO database (GSE39582). The subtypes with high or low expression levels of ZBTB7 mRNA were defined as: Low, 4.3352-6.1425; and high, 6.1433-7.1295. Cell line data were from GEO (GSE36133). Two subtypes of cell lines were defined (C1 and $\mathrm{C} 2$ ) according to the genetic signature, including epithelial-mesenchymal transition (EMT)-associated genetic expression, $\mathrm{NF}-\kappa \mathrm{B}$ signaling pathway, ABCG family and apoptosis-associated genes.

Unsupervised subtype identification. Unsupervised clustering was performed using the Brunet algorithm from the $\mathrm{R}$ package non-negative matrix factorization (NMF), based on most variant probe sets $(n=1,000)$ of chemotherapy resistance associated pathway genes in Kyoto Encyclopedia of Genes and Genomes (KEGG; genome.jp/kegg/) in $53 \mathrm{CRCs}$ cell lines. After determining the optimal number of subtypes corresponding to high cophenetic and dispersion coefficients, the final subtype assignment was regenerated for this number of subtypes, using 200 runs.

Gene set enrichment analysis. Gene set enrichment analysis was performed using java GSEA Desktop Application (Broad Institute, Cambridge, MA, USA) with the hallmark gene sets $(n=50)$ and KEGG gene sets $(n=186)$ implemented in Molecular Signatures Database (v 5.1), expression data and phenotype data were formatted following user guide, samples were permutated with NMF clustering subtype or ZBTB7 expression level for 1,000 times.

Cell lines and reagents. Human SW620 (CCL-227), SW480 (CCL-228), LoVo (CCL-229), HCT-116 (CCL-247), and HT-29 (HTB-38) CRC cell lines were cultured in Dulbecco's modified Eagle's medium (DMEM; Gibco; Thermo Fisher Scientific, Inc., Waltham, MA, USA) supplemented with $10 \%$ fetal bovine serum (Gibco; Thermo Fisher Scientific, Inc.). FHC colon epithelial cell was cultured in DMEM:F12 medium supplemented with $10 \%$ fetal bovine serum. All the cell lines were purchased from the American Type Culture Collection (Manassas, VA, USA), and were cultured at $37^{\circ} \mathrm{C}$ in $5 \% \mathrm{CO}_{2}$ atmosphere. Spheroids of cells were formed using a $3 \mathrm{D}$ culture technique $(25,26)$. As described previously (27), cells were seeded in 24-well plates coated with $2 \%$ SeaPlaque agarose (BioWhittaker ${ }^{\mathrm{TM}}$; Lonza Group, Ltd., Basel, Switzerland) in DMEM with $5 \times 10^{4}$ cells/ well in $500 \mu$ l DMEM. 5-FU was purchased from Selleck Chemicals (Houston, TX, USA). NF- $\kappa$ B inhibitor SN50 was purchased from MedChemExpress (Shanghai, China) and used at the concentration at $18 \mu \mathrm{mol} / 1$ for the indicated times.

Construction of overexpression vector and short hairpin RNA (shRNA) treatment. A ZBTB7 overexpression vector and shRNA system was constructed to explore the function and mechanism. pcDNA3.1-ZBTB7A was generated by inserting the coding region (116-1,870 bp) from SW620 cells into pcDNA3.1 vector, using HindIII restriction enzyme digestion and ligation (Invitrogen; Thermo Fisher Scientific, Inc.) The procedure was performed according to previous research (19). The resulting plasmid was used to express ZBTB7. Plasmid $(4 \mu \mathrm{g})$ was transfected into cells at $50-70 \%$ confluence using Lipofectamine $2000^{\circledR}$. ZBTB7 primer pairs were 5'-CTTAAG CTTGCCACCATGGCCGGCGGCGTGG-3' and 5'-GTCAA GCTTTTAGGCGAGTCCGGCTGTGAAGTTAC-3'. The shRNA sequences used were as follows: Control shRNA, forward CGCGAATTCACCATGGCCGGCGGCGTGG, reverse TGGCTCGAGTTAGGCGAGTCCG GCTGTGAAGT TAC; ZBTB7 shRNA, forward GATCCCGCCCACAACTA CGACCTGAATTGATATCCGTTCAGGTCGTAGTTGTGG GTTTTTTCCAAA, reverse AGCTTTTGGAAAAAACCCA CAACTACGACCTGAACGGATATCAATTCAGGTCGTAG TTGTGGGCGG. The subsequent experiments were performed $48 \mathrm{~h}$ after the transfection.

Transwell assay. The chambers (BD Biosciences, San Jose, CA, USA) were precoated with Matrigel (BD Biosciences, San Jose, CA, USA). HCT116 cells $\left(5 \times 10^{6}\right)$ and ZBTB7shRNA cells were counted and suspended in $100 \mu \mathrm{l} \mathrm{FBS-free}$ medium at the upper chamber. Medium containing 10\% FBS was added in the lower chamber, and incubated for $24 \mathrm{~h}$ at the temperature of $37^{\circ} \mathrm{C}$. Cells were fixed with $90 \%$ ethanol for $1 \mathrm{~h}$ at $4^{\circ} \mathrm{C}$. Migrant cells on membranes were visualized with $0.1 \%$ crystal violet staining for $15 \mathrm{~min}$ at room temperature. Following drying, the migrant cells were counted in five x200 microscopic fields.

In vitro chemosensitivity assay. Cell Counting Kit-8 (CCK-8; Dojindo Molecular Technologies, Inc., Kumamoto, Japan) to determine the $\mathrm{IC}_{50}$ values of cells. Following trypsinization, the transfected cell suspensions (control shRNA, ZBTB7shRNA and ZBTB7-OE) were transferred and dispersed into 96-well plates. The density was $\sim 5,000$ cells/well. 5-FU was added to treatment groups. After $72 \mathrm{~h}$, cells were incubated with $10 \mu \mathrm{l}$ CCK- 8 reagents for another $2 \mathrm{~h}$, then detected using a microplate reader at $450 \mathrm{~nm}$ absorbance (Bio-Rad Laboratories, Inc., Hercules, CA, USA).

Mouse xenografts models. Control HCT116 cells $\left(\sim 5 \times 10^{6}\right)$ and ZBTB7-shRNA cells were subcutaneously implanted into the posterior flank of BALB/c nude mice (male, 6 weeks old, purchased from Beijing Vital River Laboratory Animal Technology Co., Ltd. (Beijing, China). A total of 12 nude mice were used with 3 mice in each group. Tumor size was measured every 3 days and recorded by using the following equation: Volume $\left(\mathrm{mm}^{3}\right)=($ length $\mathrm{x}$ width2)/2. When the tumors reached $100 \mathrm{~mm}^{3}, 5-\mathrm{FU}$ was administered to the mice. Intraperitoneal injection of 5-FU was administered at a concentration of $25 \mathrm{mg} / \mathrm{kg}$ every 3 days. After $\sim 1$ month, the mice were sacrificed. The tumor tissues were extracted and the volume and weight were calculated. The maximum tumor volume was 4,200 $\mathrm{mm}^{3}$. Ethics approval for the animal experiments was provided by The Institutional Animal Care and Use Committee of Chongqing Fuling Central Hospital (Chongqing, China; ethics approval no. 2014015). 
Western blotting. The cells were lysed in radioimmunoprecipitation assay lysis buffer (Thermo Fisher Scientific, Inc.) and cell lysates were collected. Then bicinchoninic acid method was used to detect the protein concentration. Equal amounts of protein $(20 \mu \mathrm{g})$ was separated by $10 \%$ SDS-PAGE (Thermo Fisher Scientific, Inc.) and then the proteins were transferred onto polyvinylidene difluoride (PVDF) membranes (EMD Millipore, Billerica, MA, USA). Subsequently, the membranes were blocked at room temperature with 5\% bovine serum albumin (Thermo Fisher Scientific, Inc.) [BSA; in TBS-Tween (TBST)] for $1 \mathrm{~h}$ and incubated at $4^{\circ} \mathrm{C}$ with the indicated antibodies overnight. TBST was used to wash the PVDF membranes three times and incubated with secondary antibodies, anti-mouse $\operatorname{IgG}$ (cat. no. 7076; Cell Signaling Technology, Inc., Danvers, MA, USA), anti-rabbit IgG (cat. no. 7074; Cell Signaling Technology, Inc.; both diluted at 1:1,000) for $1 \mathrm{~h}$ at room temperature. TBST was used to wash again three times. Finally, proteins were observed with a chemiluminescence kit (Thermo Fisher Scientific, Inc.) by using ImageQuant LAS 4000 (GE Healthcare Life Sciences, Little Chalfont, UK). The following primary antibodies were used: GAPDH (1:3,000; Cell Signaling Technology, Inc.A; cat. no. 5174), ZBTB7 (1:1,000; Santa Cruz Biotechnology, Inc., Dallas, TX, USA; cat. no. sc-33683), E-cadherin (1:1,000; Cell Signaling Technology, Inc.; cat. no. 14472), E-selectin (1:1,000; Santa Cruz Biotechnology, Inc.; cat. no. sc-18852), integrin $\beta 1$ (1:1,000; Cell Signaling Technology, Inc.; cat. no. 34971), integrin $\alpha \mathrm{V}$ (1:500; Santa Cruz Biotechnology, Inc.; cat. no. 4711) and fibronectin (1:1,000; Santa Cruz Biotechnology, Inc.; cat. no. sc-69681).

Immunofluorescence assay. HCT116 control or HCT116shRNA cells were cultured overnight with or without 5 -FU $(50 \mu \mathrm{M})$ to reach $80-90 \%$ confluence. Then, the cells were cultured in complete medium for another $48 \mathrm{~h}$, and fixed in $4 \%$ paraformaldehyde for $10 \mathrm{~min}$ at room temperature. The cells were permeabilized in PBS containing $0.2 \%$ Triton X-100 for $5 \mathrm{~min}$ at room temperature, washed three times in TBST and blocked with 5\% BSA for $30 \mathrm{~min}$ at room temperature. The cells were incubated with antibody against cleaved caspase-3 (1:400; Cell Signaling Technology, Inc.; cat. no. 9661), ZBTB7 (1:100; Santa Cruz Biotechnology, Inc.; cat. no. sc-33683) overnight at $4^{\circ} \mathrm{C}$. After washing, the cells were labeled with $5 \mu \mathrm{g} / \mathrm{ml}$ Alexa Fluor 488 -conjugated secondary antibody at $4^{\circ} \mathrm{C}$ for 30 min (cat. no. A-10631; Thermo Fisher Scientific, Inc.), followed by examination under a fluorescence microscope (Nikon Corporation, Tokyo, Japan). DAPI was purchased from Sigma-Aldrich (Merck KGaA, Darmstadt, Germany) and was incubated at room temperature for $10 \mathrm{~min}$ at a concentration of $1 \mu \mathrm{g} / \mathrm{ml}$. Five randomly selected fields at a magnification of $\times 200$ were counted in each slide. The experiment was repeated for three times.

High-performance liquid chromatography (HPLC) analysis of bases released from the DNA of FU-treated cells. HPLC assay was performed to measure the intracellular 5-FU level in the cells. The methods for HPLC assay were performed according to a standard procedure (28). Exponentially growing cells $\left(\times 10^{7}\right)$ were treated with FU-2- ${ }^{14} \mathrm{C}\left(53 \mathrm{mCi} \mathrm{mmol}^{-1}\right.$; Sigma-Aldrich;
Merck KGaA), treated with non-radiolabeled FU and labeled with uracil-2-14C (52 $\mathrm{mCi} \mathrm{mmol}^{-1}$; Sigma-Aldrich; Merck KGaA), or treated with non-radiolabeled FU. The concentration of FU (and uracil) used was $10 \mu \mathrm{mol} / 1$ and cells were exposed to $\mathrm{FU}$ for $48 \mathrm{~h}$ (less than two cell divisions). Genomic DNA was isolated from the cells (Wizard Genomic DNA purification kit; Promega Corporation, Madison, WI, USA), and was precipitated with ethanol and the supernatant analyzed by high-performance liquid chromatography (HPLC). Fractions were collected at 0.5 -min intervals, and released DNA bases detected by scintillation counting or UV absorbance at $254 \mathrm{~nm}$; reference compounds were detected by UV absorbance.

Statistical analysis. The statistics analyses were performed using SPSS 20.0 software package (IBM Corp., Armonk, NY, USA). At least three independent experiments were performed to calculate the results presented as the mean \pm standard deviation. For comparison between two groups, two-tailed Student's t-test was performed. Multiple group comparisons were performed with one-way analysis of variance. Least significant difference was used as a post hoc test. $\mathrm{P}<0.05$ was considered to indicate a statistically significant difference.

\section{Results}

ZBTB7 was upregulated in CRC and promoted CRC progression. In order to explore genetic initiation involved in CRC, ZBTB7 expression was analyzed in CRC cell lines and tissue. ZBTB7 was upregulated in CRC cells lines compared with a normal colon epithelial cell line, and HCT116 cell had a relatively high ZBTB7 level (Fig. 1A). ZBTB7 function was evaluated in HCT116 cell lines. Transwell assay demonstrated that ZBTB7 shRNA reduced the invasion of HCT116 cells $(162.7 \pm 34.37)$ compared with the control group $(530.3 \pm 60.73$; $\mathrm{P}<0.05$; Fig. 1B). Furthermore, three-dimensional cultures of cells were performed to mimic the complex in vivo tumor microenvironment. Immunofluorescence showed control RNA cells and ZBTB7 shRNA cells (Fig. 1C). The results showed the same trends (Fig. 1D).

Silencing ZBTB7 increases the 5-FU sensitivity in $C R C$. To explore the functional role of ZBTB7 in 5-FU resistance, $\mathrm{IC}_{50}$ values were determined in ZBTB7-shRNA HCT116 cells and control groups cells by detecting cell viability in both cell lines at different concentration of 5-FU and over time. Compared with the control group, the $\mathrm{IC}_{50}$ values were significantly reduced in the ZBTB7-shRNA group. As the 5-FU concentration and time increased, the cell viability was decreased, with ZBTB7-shRNA group decreased more than the control $(\mathrm{P}<0.05$, Fig. 2A-C). By contrast, overexpression of ZBTB7 in SW480 cells (relatively low ZBTB7 level compared with other CRC cell lines; Fig. 1A) exhibited the opposite effects $(\mathrm{P}<0.05$; Fig. 2D-F). Cleaved caspase-3 is an apoptotic protein marker, and immunofluorescence demonstrated that 5-FU-induced apoptosis was increased in ZBTB7-shRNA cells compared with the control group $(\mathrm{P}<0.05$; Fig. $3 \mathrm{~A})$. Furthermore, a xenograft mouse model demonstrated that the size of tumors was reduced in the ZBTB7-shRNA group following treatment 
A

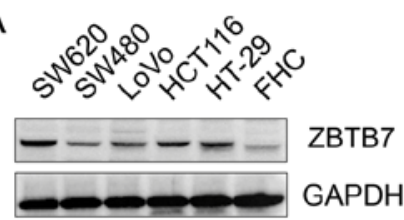

B

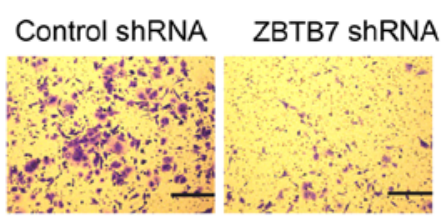

HCT116

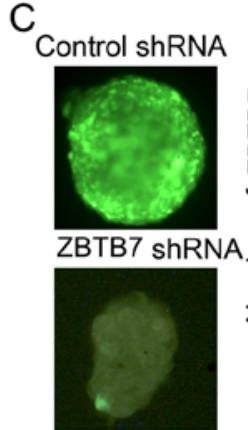

Three dimensional culture

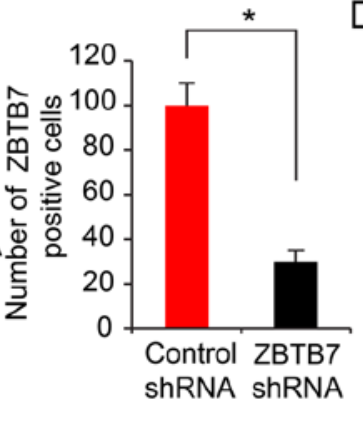

HCT116
D

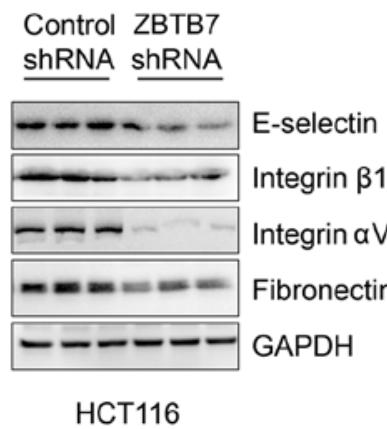

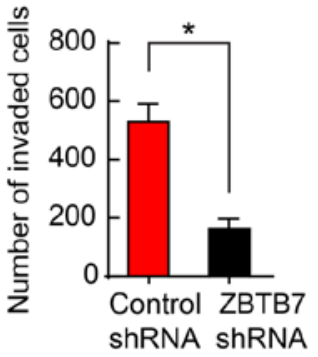

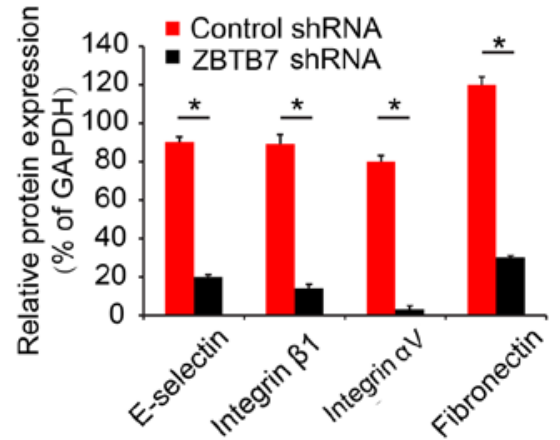

Figure 1. Expression and function of ZBTB7 in CRC. (A) Western blot analysis to measure ZBTB7 expression in CRC and normal epithelial cells lines. (B) Transwell assay of ZBTB7-shRNA and control group. ZBTB7-shRNA group showed less migrated cells than control group. (C) Representative immunofluorescence images of 3D culture of control shRNA and ZBTB7 shRNA cells. (D) Western blot analysis of relative protein expression in 3D culture. The column presents the relative protein expression " $\mathrm{P}<0.05$. CRC, colorectal cancer; ZBTB7, zinc finger and BTB domain containing 7A; shRNA, short hairpin RNA.

A

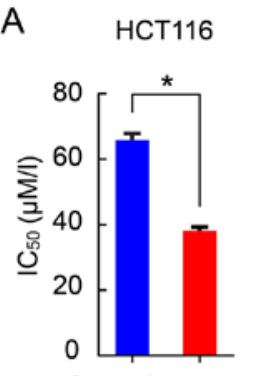

Control ZBTB7 shRNA shRNA

D

SW480

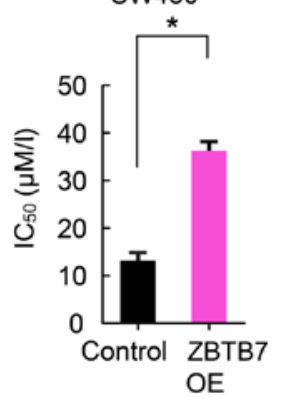

B

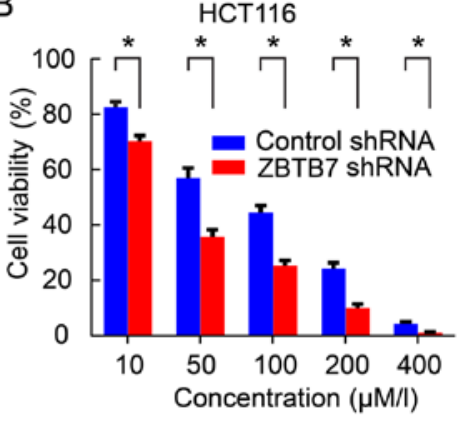

$\mathrm{E}$

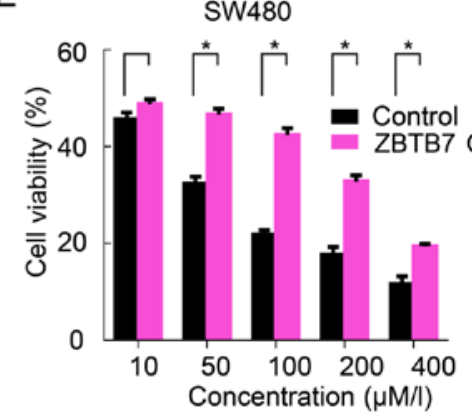

C

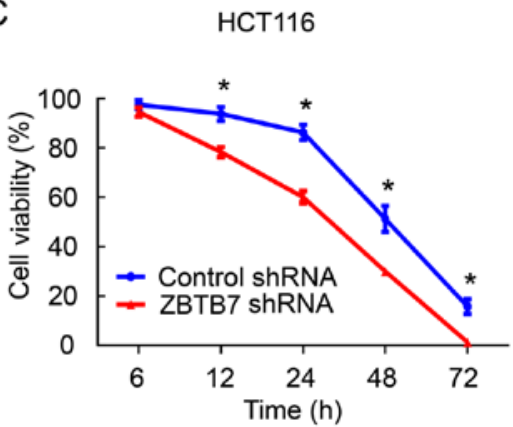

$\mathrm{F}$

SW480

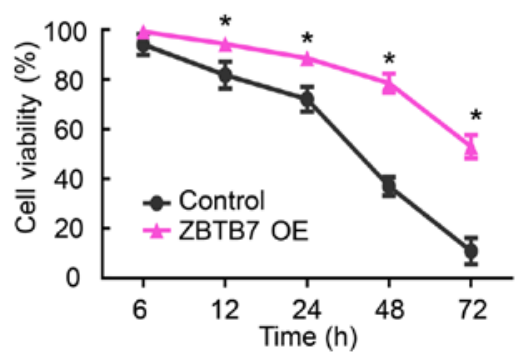

Figure 2. Silencing ZBTB7 restores the sensitivity of colorectal cancer to 5-FU. (A) CCK-8 method was measured IC 50 values in control or ZBTB7-shRNA groups exposed to 5-FU. (B) Cell viability in control or ZBTB7-shRNA groups exposed to different 5-FU concentration (10, 50, 100,200 and $400 \mu \mathrm{M})$.

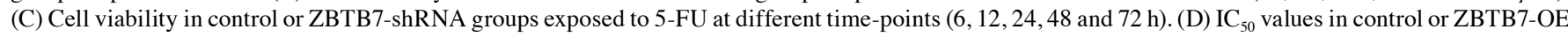
groups (SW480) exposed to 5-FU. (E) Cell vitality in control or ZBTB7-OE groups (SW480) exposed to different 5-FU concentration (10, 50, 100, 200 and $400 \mu \mathrm{M}$ ). (F) Cell viability in control or ZBTB7-OE groups (SW480) exposed to 5-FU at different time-points (6, 12, 24, 48, and 72 h). ${ }^{*}<0.05$ vs. control. 5-FU, 5-fluorouracil; shRNA, short hairpin RNA; ZBTB7, zinc finger and BTB domain containing A; OE, overexpression.

with 5-FU (Fig. 3B). The tumors volume were calculated and recorded. ZBTB7 shRNA cell-derived tumors and 5-FU were the slowest growing among all the groups $(\mathrm{P}<0.05$; Fig. 3B).

ZBTB7 is associated with $N F-\kappa B$ signaling pathways. Subsequently, it was aimed to uncover the potential mechanism of ZBTB7-driven 5-FU resistance. The original genetic profiles of patients with CRC were acquired from the GEO data repository. Differences in ZBTB7 expression among multiple cancer types were assessed using data from GEO. To explore the potential role and the mechanism of ZBTB7 in regulating chemotherapeutic resistance, data from cell lines 
A A
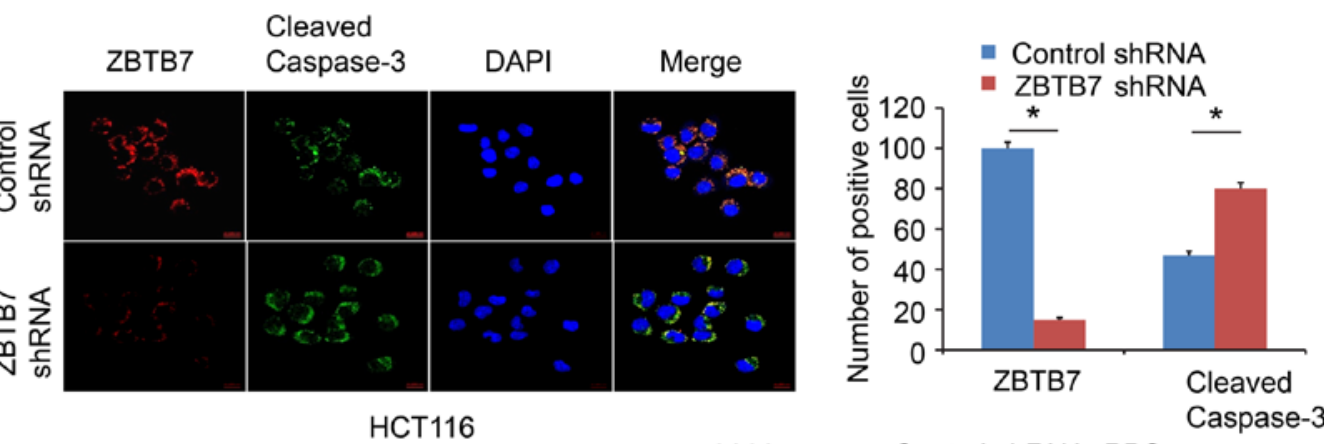

B

HCT116

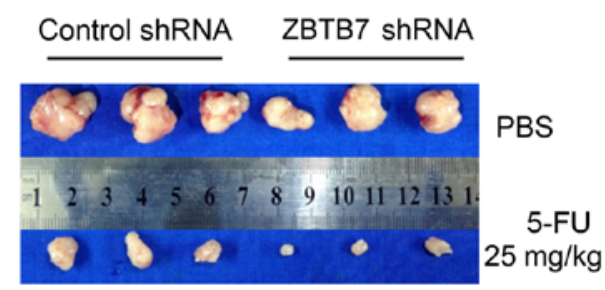

HCT116

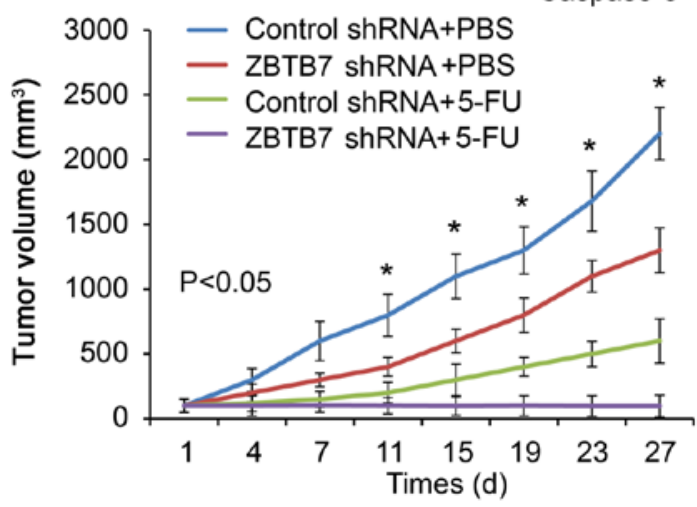

Figure 3. Effect of ZBTB7 on cell apoptosis assay and xenografts in vivo. (A) Immunofluorescence for cleaved caspase-3 and ZBTB7 expression following treatment with 5-FU in control and ZBTB7-shRNA groups. Control group (ZBTB7, 100 \pm ; cleaved caspase-3, 48 \pm 2 ), ZBTB7-shRNA group (ZBTB7, 16 \pm 2 ;

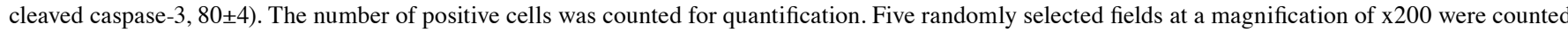
for each slide. The experiment was repeated three times. (B) Mouse xenografts tumor volume in control and ZBTB7 shRNA groups with or without 5-FU $(25 \mathrm{mg} / \mathrm{kg})$ in 27 days. " $\mathrm{P}<0.05$ vs. control group. 5-FU, 5-fluorouracil; shRNA, short hairpin RNA; ZBTB7, zinc finger and BTB domain containing 7A.

A

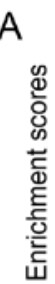

C

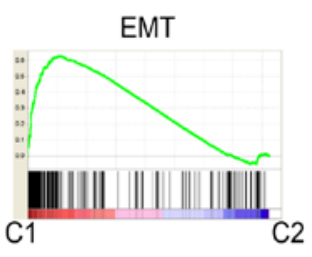

Nominal P-value 0.0163

FDR P-value 0.8323

FWER P-value 0.3930

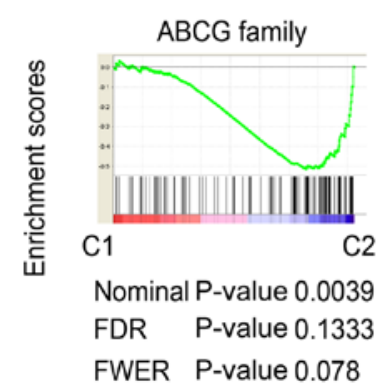

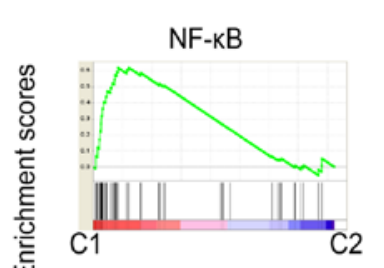

Nominal P-value 0.0423

FDR P-value 0.4254

FWER P-value 0.5140

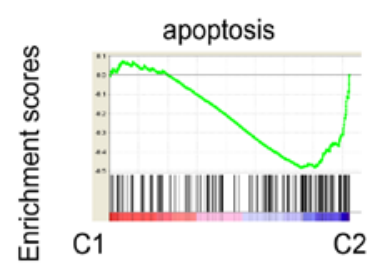

Nominal P-value 0.0039

FDR P-value 0.1381

FWER P-value 0.149
B

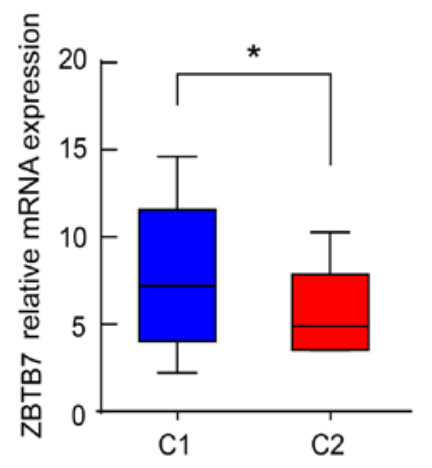

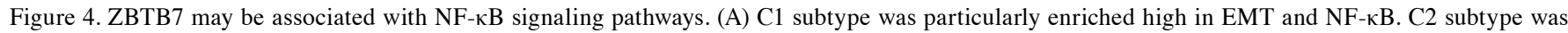
enriched in ABCG family and apoptosis. (B) The relative ZBTB7 level in group C1 was found to be significantly higher than that in group C2. Data was obtained from Gene Expression Omnibus. ${ }^{*}<<0.05$. EMT, epithelial-mesenchymal transition; FDR, false discovery rate; FWER, family-wise error rate; NF- $\kappa \mathrm{B}$, nuclear factor- $\kappa \mathrm{B}$; ABCG, ATP-binding cassette subfamily G.

(GSE36133) derived from naturally occurring tumors were analyzed because they recapitulate various aspects of the tissue type and genomic context of cancer. NMF, a recently established approach for consensus clustering, was performed onto 53 CRC cell lines according to their transcriptional features. This analysis revealed two subtypes of CRC cell lines with adequate data coherence. Bioinformatics analysis split cell lines into two subtypes according to the EMT-associated genetic expression, the NF- $\kappa \mathrm{B}$ signaling pathway, ABCG family and apoptosis-associated genes. One subtype (39.6\% of all lines, $\mathrm{n}=21$ ) was especially high in epithelial- mesenchymal transition and NF-kB (Fig. 4A), and was termed $\mathrm{C} 1$ subtype. 


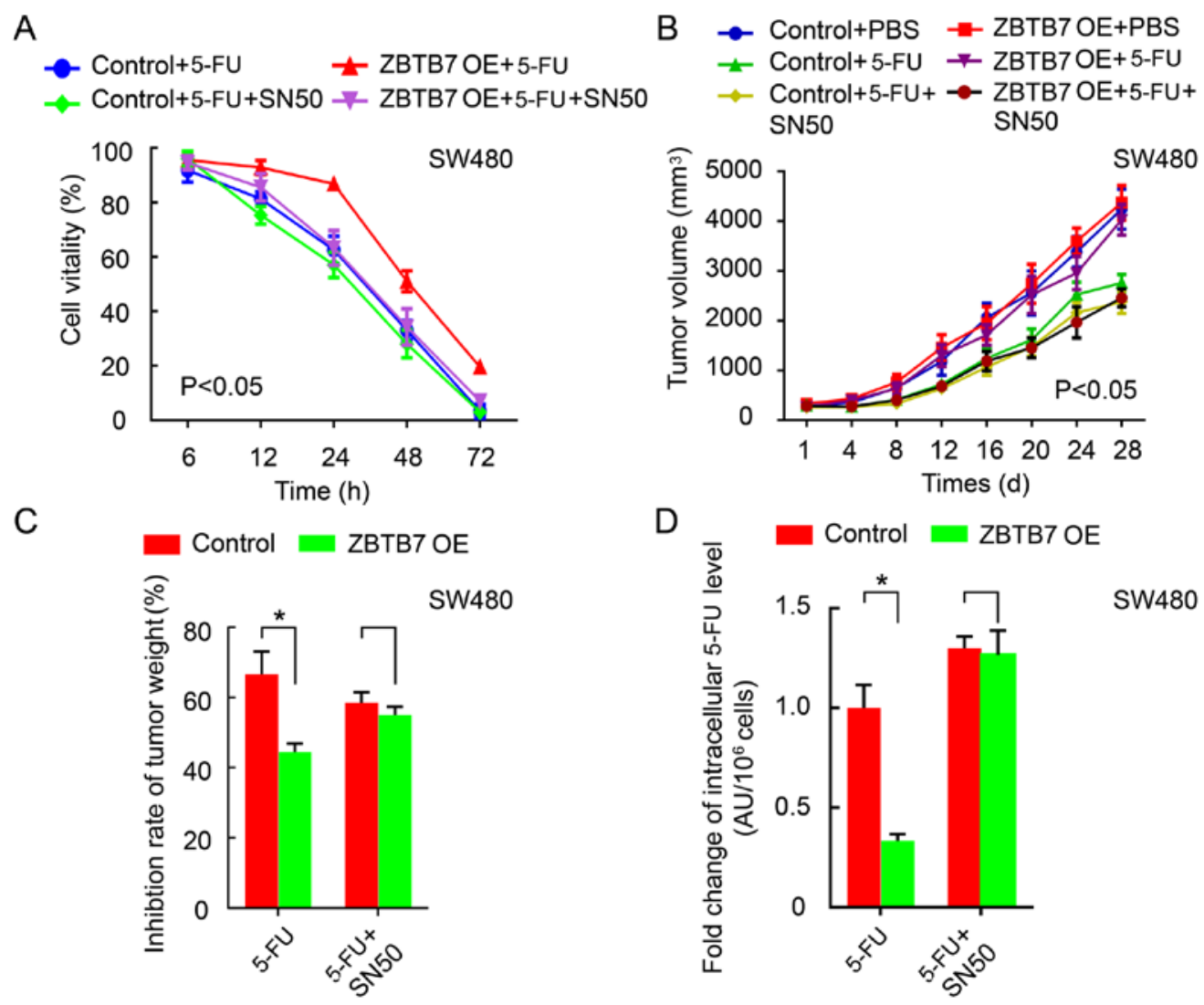

Figure 5. NF-kB inhibitor SN50 reverses ZBTB7-induced resistance in colorectal cancer cells. (A) CCK8 method was used to detect cell viability in control+5-FU with or without SN50 group, in OE+5-FU with or without SN50 group. (B) Tumor volume in control+PBS, control+5-FU, control+5-FU+SN50, in $\mathrm{OE}+\mathrm{PBS}, \mathrm{OE}+5-\mathrm{FU}, \mathrm{OE}+5-\mathrm{FU}+\mathrm{SN} 50$ group. (C) Inhibition rate of tumor weight in control+5-FU with or without SN50 group, in OE+5-FU with or without SN50 group. (D) HPLC assay was performed to measure intracellular 5-FU concentration in control+5-FU with or without SN50 group, in OE+5-FU with or

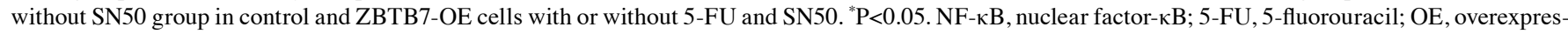
sion.
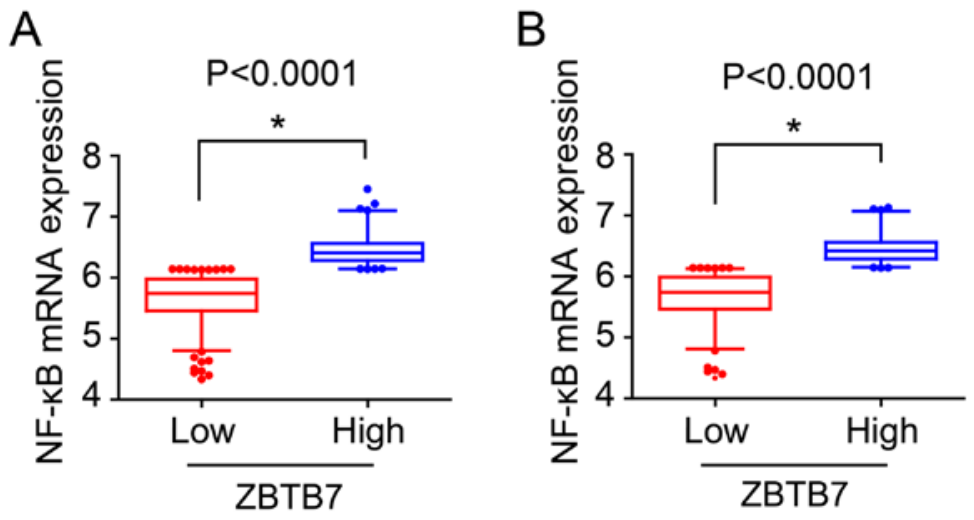

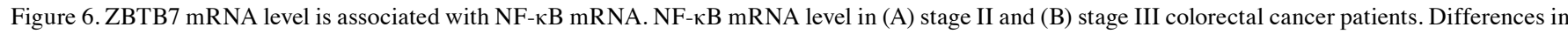

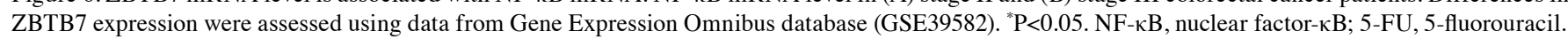

The other subtype (56.2\% of all lines, $\mathrm{n}=27$ ) especially high in ABCG family and apoptosis (Fig. 4B) were named C2 subtype. Notably, the relative ZBTB7 level in group $\mathrm{C} 1$ was significantly higher than that in group C2 (Fig. 4B). This evidence indicates that ZBTB7 may be involved in chemotherapeutic resistance of CRCs via regulation of the NF- $\mathrm{KB}$ pathway.

$N F-\kappa B$ inhibitor $S N 50$ reverses $Z B T B 7-i n d u c e d$ resistance in

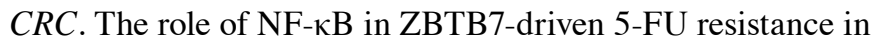

CRC was investigated. 5-FU reduced cell viability, and overexpression of ZBTB7 depleted the inhibitory effects. Treatment with $\mathrm{NF}-\kappa \mathrm{B}$ inhibitor SN50 reversed the proliferation enhancing effects of ZBTB7 ( $\mathrm{P}<0.05$; Fig. 5A). These results were verified in vivo. The tumor volume $(\mathrm{P}<0.05$; Fig. $5 \mathrm{~B})$ and weight $(\mathrm{P}<0.05$; Fig. 5C) exhibited the same trends. Finally, ZBTB7 overexpression increased the 5-FU level while SN50 significantly increased the intracellular 5-FU level $(\mathrm{P}<0.05$; Fig. 5D). 
Table I. Effect of ZBTB7 expression on overall survival and relapse-free survival of patients with stage II/III colorectal cancer according to Gene Expression Omnibus (GSE39582) clinical annotations.

A, Overall survival

\begin{tabular}{lccc}
\hline & \multicolumn{3}{c}{ Mean months (standard error) } \\
\cline { 2 - 4 } Treatment & Total & ZBTB7 Low & ZBTB7 High $^{\mathrm{a}}$ \\
\hline $5-\mathrm{FU}$ & $52.28(1.84)$ & $53.99(1.98)$ & $47.46(4.07)$ \\
$\mathrm{CC}^{\mathrm{c}}$ & $53.19(1.70)$ & $55.60(1.84)$ & $48.81(3.43)$ \\
\hline
\end{tabular}

B, Relapse-free survival

\begin{tabular}{lccc}
\hline & \multicolumn{3}{c}{ Mean months (standard error) } \\
\cline { 2 - 4 } Treatment & Total & ZBTB7 Low & ZBTB7 High $^{\mathrm{a}}$ \\
\hline $5-\mathrm{FU}$ & $43.16(2.61)$ & $46.60(2.80)$ & $32.93(5.58)$ \\
CC $^{\mathrm{c}}$ & $47.36(2.66)$ & $51.53(2.79)$ & $39.77(5.16)$ \\
\hline
\end{tabular}

${ }^{\mathrm{a} Z B T B 7}$ mRNA expression levels: low=4.3352-6.1425, high=6.1433-7.1295; ${ }^{\mathrm{b}}$ Log-rank (Mantel-Cox); ${ }^{\mathrm{c}}$ Chemotherapy regimen: Folinic acid, 5-FU and oxaliplatin; 5-FU and folinic acid; 5-FU, folinic acid and irinotecan.

ZBTB7 mRNA level is associated with 5-FU sensitivity and prognosis. The above results had demonstrated that higher ZBTB7 expression promoted tumorigenesis and susceptibility to 5-FU resistance through the NF- $\mathrm{NB}$ pathway. The clinical significance was investigated using GSE39582 from the GEO database. The patients received 5-FU alone or 5-FU-based adjuvant chemotherapy; the subgroup with ZBTB7-low tumors substantially benefited from adjuvant chemotherapy and exhibited a significantly increased probability of relapse-free survival and overall survival relative to those in the subgroup with ZBTB7-high tumors. ( $\mathrm{P}<0.05$; Fig. 6; Table I).

\section{Discussion}

5-FU-based chemotherapy is the basic classical treatment for patients with CRC (29). However, chemoresistance, either primary or acquired, is as a major challenge for clinical practice. 5-FU primary chemoresistance is predominantly due to increased thymidylate synthetase mRNA or protein level. Acquired chemoresistance mechanisms include the depletion of certain enzymes, such as thymidilate synthase, which is the target of 5-FU, or certain genetic mutations (30). Up to $40 \%$ of patients with stage II and III CRC receiving 5-FU-based adjuvant chemotherapy experience recurrence or mortality within 8 years of follow-up (31). Of patients with metastatic CRC, $50 \%$ are resistant to 5-FU-based chemotherapy $(32,33)$. It is important to investigate the 5-FU resistance mechanisms and set up therapeutic strategies to reverse the chemoresistance to improve prognosis. In the current study, ZBTB7 was overexpressed in CRC cells compared with normal colon epithelial cells. Additionally, ZBTB7 knockdown increased 5-FU sensitivity. Mechanistically, ZBTB7 potentially promoted 5-FU resistance through the $\mathrm{NF}-\kappa \mathrm{B}$ signaling pathway. Targeting NF- $\kappa \mathrm{B}$ signaling using SN50 reversed ZBTB7-mediated 5-FU resistance. Therefore, a ZBTB7/
$\mathrm{NF}-\kappa \mathrm{B}$ axis is involved in mediating 5-FU resistance in patients with CRC.

Though ZBTB7 is aberrantly overexpressed in human cancers, little is known about the mechanism that regulates this. A previous study demonstrated that the ZBTB7 gene is at the genomic locus chromosome 19p13.3, which is frequently mutated. The $\mathrm{t}(14 ; 19)(\mathrm{q} 32 ; \mathrm{p} 13.3)$ translocation is common in B-cell non-Hodgkin's lymphoma (34). ZBTB7 overexpression may aberrantly activate regulatory pathways, such as fibronectin-mediated h1-integrin ligation in precursor B leukemia cells (35). However, its function in 5-FU resistance is unknown. In the current study, ZBTB7 was upregulated in CRC cell lines compared with normal colon epithelial cells. A Transwell assay demonstrated that ZBTB7-shRNA cells were less invasive than the control group. E-selectin, integrin $\beta 1$, integrin $\alpha \mathrm{V}$ and fibronectin are adhesion molecules. Typically, cancer cells interactions initially require a selectin-mediated initial attachment and then the circulating cancer cells roll along the endothelium. Locally released chemokines activate the rolling cancer cells. This triggers integrin activation, making a firmer adhesion to cell adhesion molecules, initiating and driving the trans-endothelial migration and extravasation processes (36). In the current study, three-dimensional cultures were used to mimic the tumor microenvironment. The results revealed that integrin $\beta 1$, integrin $\alpha \mathrm{V}$ and fibronectin expression were reduced in ZBTB7-shRNA cells compared with the control group. These results indicated that ZBTB7 could regulate adhesion molecules expression in vitro and in vivo, leading to tumor progression. Furthermore, ZBTB7 knockdown and 5-FU treatment could increase apoptosis. EMT was previously reported to be associated with 5-FU resistance in pancreatic cancer (37) and lung cancer (38). We hypothesized that ZBTB7 may be involved in 5-FU resistance in CRC. Previous research demonstrated that $\mathrm{ZBTB} 7$ promoted the migration and invasion of hepatocellular carcinoma by increasing 
myocyte enhancer factor 2D expression (19). Mak et al (39) reported that ZBTB7 promoted cell migration and invasion via phosphoinositide 3-kinase/Akt signaling. Taken together, the results suggest that ZBTB7 may regulate EMT- and apoptosisassociated proteins to promote cell invasion and decrease cell apoptosis, which may mediate cell chemoresistance.

ZBTB7 was reported to be associated with efficacy of paclitaxel and cisplatin combination chemotherapy and overall survival in patients with NSCLC (40). The function of ZBTB7 in 5-FU resistance was examined in the present study. $\mathrm{IC}_{50}$ values were determined using ZBTB7-shRNA cells and control groups by measuring cell viability over different concentrations and time-points of 5-FU. The results demonstrated that the 5-FU $\mathrm{IC}_{50}$ values were reduced upon ZBTB7 depletion compared with control group, in dose- and timedependent manners. Additionally, overexpression of ZBTB7 exerted the opposite effects. The results were also validated in animal models. The volume and the weights of the tumor were lower in the ZBTB7 shRNA group following exposure to 5-FU.

GEO data was analyzed to identify genes that may be involved in the 5-FU resistance of CRC. The results demonstrated that the 5-FU resistance may be associated with the $\mathrm{NF}-\kappa \mathrm{B}$ signaling pathways. NF- $\mathrm{BB}$ signaling is reported to mediate chemoresistance in various ways $(41,42)$. Kwon et al (43) reported that gastric cancer cell resistance to 5-FU was mediated via activation of NF- $\mathrm{BB}$. ZBTB7, as an oncogene, participated in NF- $\mathrm{BB}$ signaling pathway. For instance, ZBTB7 reduces Bcl-2 expression through NF- $\mathrm{KB}$ in hepatocellular carcinoma (44). In the current study, NF- $\mathrm{KB}$ altered in ZBTB7-driven 5-FU resistance in CRC. 5-FU inhibited cell viability and overexpression of ZBTB7 depleted the inhibitory effects. NF- $\mathrm{KB}$ inhibitor SN50 reversed the proliferation enhancing effects of ZBTB7 in vitro and in vivo. Finally, clinical data was analyzed and indicated that patients with high expression of ZBTB7 have worse prognosis under 5-FU adjuvant therapy.

In conclusion, the finding indicated that the ZBTB7/NF- $\mathrm{KB}$ axis contributes to 5-FU resistance of patients with CRC, and may be serve as potential therapeutic targets to overcome 5-FU resistance.

\section{Acknowledgements}

Not applicable.

\section{Funding}

This study was supported by The Health Bureau of Chongqing (grant no. 2011-2-437).

\section{Availability of data and materials}

The datasets used and/or analyzed during the current study are available from the corresponding author on reasonable request.

\section{Authors' contributions}

ZW and XZ performed the cellular and animal studies, the statistical analysis and drafted the manuscript. WW and MZ analyzed the GEO database. YiL, YaL, JG and GX performed the cellular studies. CW and RL participated in designing the study. QZ was involved in designing the study and drafting the manuscript and revising it critically for important intellectual content and given final approval of the version to be published. All authors read and approved the final manuscript.

\section{Ethics approval and consent to participate}

Ethics approval for the animal experiments was provided by The Institutional Animal Care and Use Committee of Chongqing Fuling Central Hospital (ethics approval no. 2014015).

\section{Patient consent for publication}

Not applicable.

\section{Competing interests}

The authors declare that they have no conflicts of interests.

\section{References}

1. Siegel RL, Miller KD and Jemal A: Cancer Statistics, 2017. CA Cancer J Clin 67: 7-30, 2017.

2. Okusaka T, Funakoshi A, Furuse J, Boku N, Yamao K, Ohkawa S and Saito H: A late phase II study of S-1 for metastatic pancreatic cancer. Cancer Chemother Pharmacol 61: 615-621, 2008.

3. Morizane C, Okusaka T, Furuse J, Ishii H, Ueno H, Ikeda M, Nakachi K, Najima M, Ogura T and Suzuki E: A phase II study of S-1 in gemcitabine-refractory metastatic pancreatic cancer. Cancer Chemother Pharmacol 63: 313-319, 2009.

4. Sudo K, Yamaguchi T, Nakamura K, Denda T, Hara T, Ishihara T and Yokosuka O: Phase II study of S-1 in patients with gemcitabine-resistant advanced pancreatic cancer. Cancer Chemother Pharmacol 67: 249-254, 2011.

5. Sasaki T, Isayama H, Yashima Y, Yagioka H, Kogure $H$, Arizumi T, Togawa O, Matsubara S, Ito Y, Nakai Y, et al: S-1 monotherapy in patients with advanced biliary tract cancer. Oncology 77: 71-74, 2009.

6. Tsuji W, Ishiguro H, Tanaka S, Takeuchi M, Ueno T and Toi M: Orally administered S-1 suppresses circulating endothelial cell counts in metastatic breast cancer patients. Int J Clin Oncol 19: 452-459, 2014.

7. Saek T, Takashima S, Sano M, Horikoshi N, Miura S, Shimizu S, Morimoto K, Kimura M, Aoyama H, Ota J, et al: A phase II study of S-1 in patients with metastatic breast cancer - a Japanese trial by the S-1 Cooperative Study Group, Breast Cancer Working Group. Breast Cancer 11: 194-202, 2004.

8. Lee SJ, Lee J, Park SH, Park JO, Park YS, Kang WK, Lee J, Yim DS and Lim HY: Phase 1 trial of S-1 in combination with sorafenib for patients with advanced hepatocellular carcinoma. Invest New Drugs 30: 1540-1547, 2012.

9. Furuse J, Okusaka T, Kaneko S, Kudo M, Nakachi K, Ueno H, Yamashita T and Ueshima K: Phase I/II study of the pharmacokinetics, safety and efficacy of S-1 in patients with advanced hepatocellular carcinoma. Cancer Sci 101: 2606-2611, 2010.

10. Pectasides D, Karavasilis V, Papaxoinis G, Gourgioti G, Makatsoris T, Raptou G, Vrettou E, Sgouros J, Samantas E, Basdanis G, et al: Randomized phase III clinical trial comparing the combination of capecitabine and oxaliplatin (CAPOX) with the combination of 5-fluorouracil, leucovorin and oxaliplatin (modified FOLFOX6) as adjuvant therapy in patients with operated high-risk stage II or stage III colorectal cancer. BMC Cancer 15: 384, 2015.

11. Argilés G, Saunders MP, Rivera F, Sobrero A, Benson A III, Guillén Ponce C, Cascinu S, Van Cutsem E, Macpherson IR, Strumberg D, et al: Regorafenib plus modified FOLFOX6 as first-line treatment of metastatic colorectal cancer: A phase II trial. Eur J Cancer 51: 942-949, 2015.

12. Longley DB, Harkin DP and Johnston PG: 5-fluorouracil: Mechanisms of action and clinical strategies. Nat Rev Cancer 3: 330-338, 2003. 
13. Álvarez P, Marchal JA, Boulaiz H, Carrillo E, Vélez C, Rodríguez-Serrano F, Melguizo C, Prados J, Madeddu R and Aranega A: 5-Fluorouracil derivatives: A patent review. Expert Opin Ther Pat 22: 107-123, 2012.

14. Maeda T, Hobbs RM and Pandolfi PP: The transcription factor Pokemon: A new key player in cancer pathogenesis. Cancer Res 65: 8575-8578, 2005.

15. Davies JM, Hawe N, Kabarowski J, Huang QH, Zhu J, Brand NJ, Leprince D, Dhordain P, Cook M, Morriss-Kay G, et al: Novel $\mathrm{BTB} / \mathrm{POZ}$ domain zinc-finger protein, $\mathrm{LRF}$, is a potential target of the LAZ-3/BCL-6 oncogene. Oncogene 18: 365-375, 1999.

16. Lin CC, Zhou JP, Liu YP, Liu JJ, Yang XN, Jazag A, Zhang ZP, Guleng B and Ren JL: The silencing of Pokemon attenuates the proliferation of hepatocellular carcinoma cells in vitro and in vivo by inhibiting the PI3K/Akt pathway. PLoS One 7: e51916, 2012.

17. Zhao Z, Wang J, Wang S, Chang H, Zhang T and Qu J: LncRNA CCAT2 promotes tumorigenesis by over-expressed Pokemon in non-small cell lung cancer. Biomed Pharmacother 87: 692-697, 2017.

18. Aggarwal H, Aggarwal A and Agrawal DK: Corrigendum to 'Epidermal growth factor increases LRF/Pokemon expression in human prostate cancer cells' [Exp. Mol. Pathol. 91 (2011) 496-501]. Exp Mol Pathol 100: 361, 2016.

19. Kong J, Liu X, Li X, Wu J, Wu N, Chen J and Fang F: Pokemon promotes the invasiveness of hepatocellular carcinoma by enhancing MEF2D transcription. Hepatol Int 10: 493-500, 2016.

20. Sartini D, Lo Muzio L, Morganti S, Pozzi V, Di Ruscio G, Rocchetti R, Rubini C, Santarelli A and Emanuelli M: Pokemon proto-oncogene in oral cancer: Potential role in the early phase of tumorigenesis. Oral Dis 21: 462-469, 2015.

21. He S, Liu F, Xie Z, Zu X, Xu W and Jiang Y: P-Glycoprotein/ MDR1 regulates pokemon gene transcription through p53 expression in human breast cancer cells. Int J Mol Sci 11: 3309-051, 2010.

22. Zhao GT, Yang LJ, Li XX, Cui HL and Guo R: Expression of the proto-oncogene Pokemon in colorectal cancer - inhibitory effects of an siRNA. Asian Pac J Cancer Prev 14: 4999-5005, 2013.

23. Zhao Y, Yao YH, Li L, An WF, Chen HZ, Sun LP, Kang HX, Wang S and Hu XR: Pokemon enhances proliferation, cell cycle progression and anti-apoptosis activity of colorectal cancer independently of p14ARF-MDM2-p53 pathway. Med Oncol 31: 288, 2014.

24. de Reyniès A, Assié G, Rickman DS, Tissier F, Groussin L, René-Corail F, Dousset B, Bertagna X, Clauser E and Bertherat J: Gene expression profiling reveals a new classification of adrenocortical tumors and identifies molecular predictors of malignancy and survival. J Clin Oncol 27: 1108-1115, 2009.

25. Desoize B and Jardillier J: Multicellular resistance: A paradigm for clinical resistance? Crit Rev Oncol Hematol 36: 193-207, 2000.

26. Green SK, Francia G, Isidoro C and Kerbel RS: Antiadhesive antibodies targeting E-cadherin sensitize multicellular tumor spheroids to chemotherapy in vitro. Mol Cancer Ther 3: 149-159, 2004.

27. Zheng C, Zhou Q, Wu F, Peng Q, Tang A, Liang H and Zeng Y: Semaphorin $3 \mathrm{~F}$ down-regulates the expression of integrin alpha(v) beta3 and sensitizes multicellular tumor spheroids to chemotherapy via the neuropilin-2 receptor in vitro. Chemotherapy 55: 344-352, 2009.

28. Butler WR and Guthertz LS: Mycolic acid analysis by highperformance liquid chromatography for identification of Mycobacterium species. Clin Microbiol Rev 14: 704-726, 2001.

29. Tan BR, Thomas F, Myerson RJ, Zehnbauer B, Trinkaus K, Malyapa RS, Mutch MG, Abbey EE, Alyasiry A, Fleshman JW, et al: Thymidylate synthase genotype-directed neoadjuvant chemoradiation for patients with rectal adenocarcinoma. J Clin Oncol 29: 875-883, 2011.
30. Temraz S, Mukherji D, Alameddine R and Shamseddine A: Methods of overcoming treatment resistance in colorectal cancer. Crit Rev Oncol Hematol 89: 217-230, 2014.

31. Sargent D, Sobrero A, Grothey A, O'Connell MJ, Buyse M, Andre T, Zheng Y, Green E, Labianca R, O'Callaghan C, et al: Evidence for cure by adjuvant therapy in colon cancer: Observations based on individual patient data from 20,898 patients on 18 randomized trials. J Clin Oncol 27: 872-877, 2009.

32. Douillard JY, Cunningham D, Roth AD, Navarro M, James RD, Karasek P, Jandik P, Iveson T, Carmichael J, Alakl M, et al: Irinotecan combined with fluorouracil compared with fluorouracil alone as first-line treatment for metastatic colorectal cancer: A multicentre randomised trial. Lancet 355: 1041-1047, 2000.

33. Giacchetti S, Perpoint B, Zidani R, Le Bail N, Faggiuolo R, Focan C, Chollet P, Llory JF, Letourneau Y, Coudert B, et al: Phase III multicenter randomized trial of oxaliplatin added to chronomodulated fluorouracil-leucovorin as first-line treatment of metastatic colorectal cancer. J Clin Oncol 18: 136-147, 2000.

34. Gozzetti A, Davis EM, Espinosa R III, Fernald AA, Anastasi J and Le Beau MM: Identification of novel cryptic translocations involving IGH in B-cell non-Hodgkin's lymphomas. Cancer Res 62: 5523-5527, 2002

35. Astier AL, Xu R, Svoboda M, Hinds E, Munoz O, de Beaumont R, Crean CD, Gabig T and Freedman AS: Temporal gene expression profile of human precursor B leukemia cells induced by adhesion receptor: Identification of pathways regulating B-cell survival. Blood 101: 1118-1127, 2003.

36. Walzog B and Gaehtgens P: Adhesion molecules: The path to a new understanding of acute inflammation. News Physiol Sci 15: $107-113,2000$

37. Arumugam T, Ramachandran V, Fournier KF, Wang H, Marquis L, Abbruzzese JL, Gallick GE, Logsdon CD, McConkey DJ and Choi W: Epithelial to mesenchymal transition contributes to drug resistance in pancreatic cancer. Cancer Res 69: 5820-5828, 2009.

38. Wu D, Zhao B, Qi X, Peng F, Fu H, Chi X, Miao QR and Shao S: Nogo-B receptor promotes epithelial-mesenchymal transition in non-small cell lung cancer cells through the Ras/ERK/Snaill pathway. Cancer Lett 418: 135-146, 2018.

39. Mak VC, Wong OG, Siu MK, Wong ES, Ng WY, Wong RW, Chan KK, Ngan HY and Cheung AN: FBI-1 is overexpressed in gestational trophoblastic disease and promotes tumor growth and cell aggressiveness of choriocarcinoma via PI3K/Akt signaling. Am J Pathol 185: 2038-2048, 2015.

40. Zhang QL, Xing XZ, Li FY, Xing YJ and Li J: Pretreatment Pokemon level as a predictor of response to cisplatin and Paclitaxel in Patients with Unresectable Non-Small Cell Lung Cancer. Oncol Res Treat 38: 496-502, 2015.

41. Kim JK, Kim KD, Lee E, Lim JS, Cho HJ, Yoon HK, Cho MY, Baek KE, Park YP, Paik SG, et al: Up-regulation of Bfl-1/A1 via NF-kappaB activation in cisplatin-resistant human bladder cancer cell line. Cancer Lett 212: 61-70, 2004.

42. Wang CY, Guttridge DC, Mayo MW and Baldwin AS Jr: NF-kappaB induces expression of the Bcl-2 homologue A1/Bfl-1 to preferentially suppress chemotherapy-induced apoptosis. Mol Cell Biol 19: 5923-5929, 1999.

43. Kwon OH, Kim JH, Kim SY and Kim YS: TWEAK/Fn14 signaling mediates gastric cancer cell resistance to 5-fluorouracil via NF-אB activation. Int J Oncol 44: 583-590, 2014.

44. Zhao X, Ning Q, Sun X and Tian D: Pokemon reduces Bcl-2 expression through NF- $\kappa$ Bp65: A possible mechanism of hepatocellular carcinoma. Asian Pac J Trop Med 4: 492-497, 2011. 